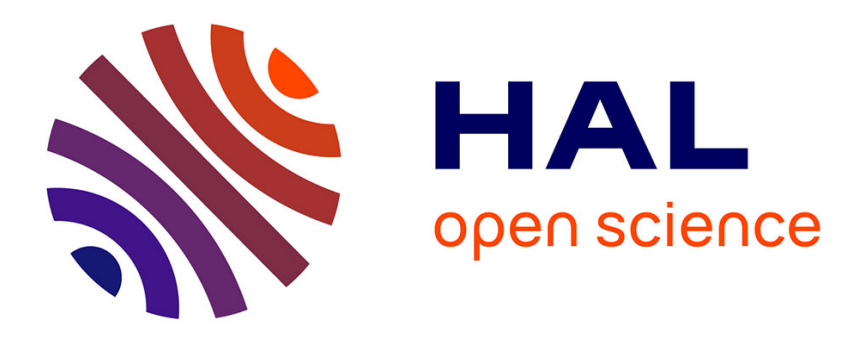

\title{
Privatisations du patrimoine public et politiques économiques
}

Philippe Bance

\section{To cite this version:}

Philippe Bance. Privatisations du patrimoine public et politiques économiques. Revue d'économie industrielle , 1988, 46 (1), pp.21-33. 10.3406/rei.1988.2235 . hal-01965198

HAL Id: hal-01965198

https://hal-normandie-univ.archives-ouvertes.fr/hal-01965198

Submitted on 25 Dec 2018

HAL is a multi-disciplinary open access archive for the deposit and dissemination of scientific research documents, whether they are published or not. The documents may come from teaching and research institutions in France or abroad, or from public or private research centers.
L'archive ouverte pluridisciplinaire HAL, est destinée au dépôt et à la diffusion de documents scientifiques de niveau recherche, publiés ou non, émanant des établissements d'enseignement et de recherche français ou étrangers, des laboratoires publics ou privés. 


\section{Privatisations du patrimoine public et politiques économiques} Philippe Bance

Citer ce document / Cite this document :

Bance Philippe. Privatisations du patrimoine public et politiques économiques . In: Revue d'économie industrielle, vol. 46, 4e trimestre 1988. pp. 21-33;

doi : https://doi.org/10.3406/rei.1988.2235

https://www.persee.fr/doc/rei_0154-3229_1988_num_46_1_2235

Fichier pdf généré le 09/04/2018 


\begin{abstract}
To reduce the theory of privatization to that of denationalization is conceptually incorrect. Beyond a simple transfert of property privatization, in its broadest sense, may be considered as a multifaceted phenomen of assimilation or even of assistance (under the aegis of the State) of public ownership to private sector.

This extended approach becomes indispensable if one attempts to achieve a bette understanding of the processes of privatization which have been developing in liberal economies since the last world war. It leads to the refutation of the theory that privatizations have been following a uniform and convergent path on the international stage. It also demontrates the existence of different periods, during which privatizations were affected by structural changes and in particular by economic policy trends.
\end{abstract}

\title{
Résumé
}

Ramener l'étude des privatisations à celle des dénationalisations relève de l'erreur conceptuelle. Audelà du simple transfert de propriété, la privatisation est, au sens large, un phénomène d'assimilation ou même de soutien (sous l'égide de l'État) du patrimoine public au secteur privé.

Cette approche élargie s'avère indispensable si l'on entend appréhender correctement les processus de privatisation qu'ont connu les économies libérales depuis l'après-guerre. Elle conduit à récuser les thèses selon lesquelles il y aurait uniformité et convergence sur le plan international. Elle permet également de mettre en lumière l'existence de phases de privatisation différenciées qu'il convient d'associer aux mutations structurelles et surtout aux orientations de politique économique. 


\title{
Privatisations du patrimoine public et politiques économiques
}

\author{
Philippe BANCE \\ Maître de Conférences \\ Université de Lille II
}

Le concept de privatisation est incontestablement devenu l'un des plus utilisé du discours économico-médiatique. Un véritable consensus de la presse écrite et audio-visuelle semble s'être dégagé pour considérer que privatisation signifie transfert de propriété (et plus encore de contrôle) du public vers le privé. Cette définition, transposition littérale du terme anglo-saxon de " privatization ", est d'ailleurs largement reprise dans le débat scientifique, et paraît aller de soi pour de nombreux auteurs. Et pourtant, n'existe-t-il pas dans le vocabulaire français un concept qui désigne sans équivoque le transfert de contrôle public-privé : celui de dénationalisation. Ne s'agirait-il alors que d'une méprise terminologique, produit de la sous-culture médiatique, mais n'ayant d'autre portée réelle que celle de la sémantique ? Certes non. Cette approche conceptuelle tend en fait à escamoter la nature même du processus de privatisation que connaissent les économies libérales depuis quelques années en en donnant une image réductrice. Ne doiton pas en effet considérer la dénationalisation comme la forme la plus radicale (souvent le point d'aboutissement terminal) d'un processus de pénétration de composantes du secteur public par le secteur privé ?

Une discussion approfondie sur le concept de privatisation s'impose donc (I). Cet éclairage conceptuel nous permettra alors d'appréhender la nature profonde des politiques de privatisation du patrimoine public qu'ont connues les économies libérales européennes et de percevoir les motivations structurelles cachées du phénomène. Pour ce faire, il s'avèrera nécessaire de repenser les privatisations en les mettant en parallèle avec l'« utilisation » qui est faite des secteurs publics dans le cadre des politiques économiques (II).

\section{I. - APPROCHE CONCEPTUELLE}

Traiter des privatisations du patrimoine public (1) consiste, au sens large du terme, à relater l'existence de caractéristiques ou d'évolutions qui l'assimile au

(1) Seule la privatisation du patrimoine public nous retiendra ici, bien qu'elle ne constitue qu'une forme (essentielle il est vrai) parmi d'autres de privatisation. La déréglementation, l'extension des formules d'allocations ou de concession au privé relèvent également des privatisations. Concernant les déréglementations voir notamment : J. BARREAU et A. MOULINE, " La déréglementation américaine des télécommunications et l'Europe ", Revue d'économie industrielle, $\mathrm{n}^{\circ} 39$,

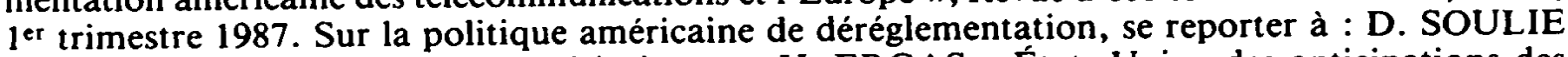
"Télécommunications : leçon américaine » et H. ERGAS "États-Unis : des anticipations des économistes aux résultats sur le terrain ", Revue française de gestion. F. ESCARMELLE et J. HUJOEL "Privatization and regulation". Its implementation in Belgium. Annales de l'économie publique, sociale et coopérative, précité, ce dernier article traite également du cas belge. 
patrimoine privé ou le soumettent aux intérêts privés. Cette définition générale appelle plusieurs remarques.

Les privatisations peuvent fondamentalement être appréhendées sur deux plans. Etudier les modalités d'assimilation ou de soumission du patrimoine public vis-àvis du secteur privé apparaissant à un moment donné revient à percevoir le phénomène comme un état, une caractéristique observée, alors qu'une analyse en termes d'évolutions (en particulier en termes d'extension de pratiques organisées) consiste à envisager les privatisations comme un processus. Nous opterons ici pour cette seconde approche.

La définition que nous donnons du concept de privatisation repose par ailleurs sur l'idée que le patrimoine public - et nous nous intéressons tout particulièrement au secteur public productif - est doté de spécificités comportementales qui, le cas échéant, le démarquent du patrimoine privé. Cette "distanciation potentielle ", qui fonde en fait les privatisations, se situe à deux niveaux.

Elle concerne tout d'abord les exigences de rémunération. Alors que les agents privés cherchent, en toutes circonstances, à retirer le profit maximal de leur patrimoine productif, l'État peut y renoncer pour faire remplir par ses capitaux des missions d'animation et de régulation de l'activité économique (2). Ce renoncement n'est nullement permanent ou généralisé, le management public prônant, afin de s'émanciper de l'État-propriétaire, l'adoption de comportements stratégiques tendus par la mise en valeur maximale. C'est dire que les entreprises publiques (que l'on définira comme des sociétés contrôlées de manière directe ou indirecte par l'État) peuvent se comporter comme des entreprises privées (3). Leur comportement est alors privatisé. Par contre, l'infléchissement par l'État de la fonction objectif des entreprises publiques publicise leurs comportements. Cette publicisation comportementale est cependant, elle-même, à l'origine d'un autre type de privatisation lorsqu'elle consacre des transferts de ressources public-privé (4).

La distanciation comportementale public-privé concerne également le pouvoir de contrôle sur les entreprises. Alors que les agents privés exercent toujours ce pouvoir lorsqu'ils sont en position de le faire, ce n'est pas forcément le cas de la puissance publique. Celle-ci peut y renoncer - ou amener des entreprises publiques à y renoncer - alors que ses titres de propriété sont quantitativement suffisants pour l'exercer (5). Elle peut également décider de réduire sa présence dans

(2) Cf. L. MONNIER, Capitaux publics et stratégie de l'État, PUF, 1977, pp. 183-188.

(3) Nous considérons que les entreprises privées cherchent de manière permanente à maximiser leur profit. La présence d'un pouvoir managérial n'y change rien à notre sens, pensant avec Y. MORVAN qu'il y a alors identité entre maximisations de la croissance et du profit. Cf. Y. MORVAN, Influence de la dimension sur la rentabilité des entreprises industrielles, Thèse, Rennes, 1967, pp. 644-649.

(4) Il faut entendre ici par transferts l'évaluation monétaire des différents moyens d'intervention s'appliquant aux agents économiques. Cf. A. LEPORS, « Les transferts État-industrie en France et dans les pays occidentaux ", Notes et études documentaires, $n^{\circ} 4303-05$, juil. 1976.

(5) Cf. P. BANCE, «Sur l'approche matricielle des rapports de propriété et la théorie du contrôle ", Revue économique, vol. $39, \mathrm{n}^{\circ} 2$, mars 1988. 
l'économie afin, principalement, de favoriser l'expansion du secteur privé. Ces comportements publics spécifiques de non maximisation du contrôle fondent en fait les privatisations par transferts d'actifs.

Il convient cependant d'être plus explicite en présentant les principales modalités institutionnelles de privatisation. Nous allons, ce faisant, marquer l'opposition entre subordination (A) et assimilation (B) du patrimoine public.

\section{A - Privatisations par différenciation subordonnée}

Ce type de privatisation, qui concerne la fonction d'assistance du secteur public au développement du secteur privé, relève 1) de transferts de ressources financières ou 2) de " transferts » en capital.

\section{Les transferts tarifaires et commerciaux}

Le contrôle exercé par l'État sur les tarifs des entreprises publiques (monopolistiques en particulier) est fréquemment à l'origine de retards dans les ajustements de prix. Au-delà de leur caractère anti-inflationniste, ces compressions tarifaires (6) alimentent les profits des entreprises du système productif (donc des sociétés privées) en réduisant leurs coûts ou en leur octroyant des avantages comparatifs eu égard aux firmes étrangères. Il y a alors privatisation de ressources financières publiques afin de générer des " effets d'environnement ".

Les transferts peuvent également provenir de relations commerciales particulières entre entreprises publiques et privées. C'est notamment le cas lorsque des marchandises ou services sont - au regard des prix de marché ou des coûts vendus à bas prix par les entreprises publiques aux entreprises privées, ou inversement, à prix élevés par des sociétés privées à leurs homologues publics. De tels transferts, à caractère ponctuel, s'opèrent bien souvent par l'intermédiaire des sociétés mixtes, ces dernières constituant un lieu d'expression privilégié des relations commerciales discriminatoires public-privé (7).

\section{Les transferts en capital}

Les cessions d'actifs publics peuvent également conduire à des transferts de ressources financières vers le secteur privé. Ainsi, les politiques de dénationalisation massive sont souvent réalisées à des prix de vente des actifs se situant en deçà de leur valeur effective (8). Plus largement, les cessions d'actifs publics permettent le positionnement (voire assurent le redéploiement) des capitaux privés sur les activités publiques rentables (9).

(6) Nous parlerons de compressions tarifaires lorsque l'évolution des tarifs des entreprises publiques se situe en deçà de l'évolution de leurs coûts.

(7) Voir, pour la France, P. BANCE et L. MONNIER, « The privatization of public enterprises in France ", The privatization of public enterprises, Special issue, Annales de l'économie publique, sociale et coopérative, 1986, pp. 57-59.

(8) Ce fut le cas en Grande-Bretagne et en France sur les dernières années : l'évolution du cours des actions aussitôt après la souscription des titres est à cet égard significative.

(9) Cf. P. BANCE, Participations publiques et politique industrielle, Thèse de $3^{e}$ cycle, Rouen, 1984, pp. 122-128. 
Au-delà des transferts de ressources financières associées au cessions d'actifs, les transferts de contrôle (ou renonciations publiques au contrôle) constituent une autre modalité de privatisation par différenciation subordonnée. Il s'agit en effet de stratégies publiques spécifiques ayant pour objet de dynamiser le secteur privé en lui concédant l'« usage » des capitaux publics. Le baillage de fonds publics, c'est-à-dire la diffusion ultra-minoritaire de capitaux publics dans les unités du système productif, constitue un second volet de cette même stratégie.

\section{B - Privatisations par assimilation}

L'assimilation des entreprises publiques à celles du secteur privé peut 1) être comportementale et/ou 2) s'exprimer juridiquement (la transmutation).

\section{L'assimilation comportementale}

L'assimilation comportementale est la tendance à l'alignement des comportements des unités publiques sur les critères de gestion privés. Elle concerne donc toute extension de la place prise par le mobile de mise en valeur maximale dans leur fonction objectif au détriment du mobile régulationniste de la puissance publique. Impulsée par des directions d'entreprises publiques à la recherche d'indépendance, l'assimilation comportementale peut s'imposer aux autorités pour plusieurs raisons de fond concomitantes et synergiques. L'ouverture croissante des économies libérales, l'intensification de la concurrence, la crainte (ou la volonté de réduire) des déficits sont ici à mettre en exergue.

Cependant, la transformation des structures organisationnelles ou patrimoniales constitue souvent le préalable ou le corollaire de cette assimilation comportementale.

\section{La transmutation des capitaux publics}

Par transmutation des capitaux publics, il faut entendre toute modification des modalités d'exercice de l'activité productive publique pour les rapprocher de celles qui sont en usage dans la sphère privée. Présentons en les principales variantes par ordre de radicalité croissante : les modifications statutaires (a), la filialisation (b), la dénationalisation (c).

\section{a) Les mutations statutaires}

Les entreprises publiques de premier rang sont fréquemment dotées, surtout lorsqu'elles se situent en secteur monopolistique, de statuts régissant strictement le contenu de leurs activités et fixant des contraintes rigoureuses (10) qui obligent en fait l'État à couvrir les pertes éventuelles.

Ces rigidités d'ordre juridique constituent bien entendu des entraves à l'assimilation comportementale voire à la « responsabilisation » des directions et des personnels d'entreprises publiques. Des modifications statutaires, et notamment

(10) Cf. J. DUFAU « Les entreprises publiques », Editions de l'actualité juridique, 1973. 
l'adoption du régime juridique des sociétés par actions, peuvent alors apparaître aux autorités comme une réponse appropriée. Elles constituent même parfois un préliminaire à la dénationalisation.

\section{b) La filialisation}

Le tissage de liens financiers, la constitution de filiales et de participations par les entreprises publiques - que l'on peut taxer de filialisation publique - peuvent être à l'origine de profonds bouleversements dans la configuration patrimoniale publique, la rapprochant de celle du secteur privé. Des entreprises publiques monolithiques ont ainsi pu se transformer en puissants groupes ramifiés et diversifiés. Il s'agit en fait de la résultante juridico-structurelle la plus tangible de l'assimilation comportementale.

Le rapprochement des structures productives publiques et privées peut d'ailleurs conduire à des associations dans le cadre sociétaire : par constitution de sociétés mixtes ou encore par cession partielle du capital des entreprises publiques au secteur privé. Les directions d'entreprises publiques y voient alors souvent le moyen de se prémunir contre d'éventuelles injonctions de la puissance publique, la présence des capitaux privés rendant pour le moins délicate la publicisation de la fonction objectif des sociétés considérées (11).

\section{c) La dénationalisaton}

La dénationalisation est le passage d'actifs publics dans le secteur privé du fait d'une politique délibérée (12) d'un État désirant réduire le poids du secteur public. Il s'agit donc de la forme extrême de l'assimilation puisque, une fois l'opération réalisée, l'entreprise (ou les actifs physiques) considérés est privée tant dans ses comportements que dans sa propriété. Il convient cependant de distinguer les approches juridiques et économiques du concept de dénationalisation.

Au sens juridique, la dénationalisation concerne toute opération, orchestrée par l'État, de vente d'actifs publics physiques au privé ou de cession de titres de propriété conférant aux agents privés la majorité du capital social dans des entreprises précédemment publiques. Si cette approche recouvre la plupart des opérations concrètes de dénationalisation, elle n'en est pas moins réductrice. Les transferts de contrôle qui s'effectuent sans cession de titres de propriété ne sont pas pris en considération bien qu'aboutissant à un passage sous le giron privé. $\boldsymbol{A}$ contrario, on peut considérer que l'approche juridique est excessive car l'abandon public de la détention d'une majorité de capital social ne conduit pas obligatoirement au contrôle privé, l'existence d'une pluralité d'actionnaires privés pouvant permettre le maintien du contrôle public avec participation minoritaire. Autant de raisons nous incitant à retenir la définition économique de la dénationalisation : le passage d'actifs ou d'unités d'un contrôle public à un contrôle privé.

(11) La mixité peut néanmoins aboutir à des relations discriminatoires entre participants publics et privés des sociétés mixtes (cf. supra).

(12) Nous ne faisons donc pas relever de la dénationalisation les cessions d'actifs auxquelles procèdent les directions d'entreprises publiques dans le cadre de réorientations stratégiques, comme le feraient leurs homologues privés. Ces opérations ont en effet pour logique ultime l'adaptation du secteur public à l'environnement et non la cession au secteur privé. 
Cet éclairage conceptuel va maintenant nous permettre d'étudier avec exactitude le processus de privatisation dans les pays européens.

\section{II. - ANALYSE RÉTROSPECTIVE DES PRIVATISATIONS EUROPÉENNES}

Dresser un cadre général d'interprétation du processus de privatisation du patrimoine public qu'ont connu les économies libérales européennes durant les dernières décennies peut sembler, de prime abord, une gageure. Le caractère multidimensionnel du concept de privatisation, l'apparente diversité, tant dans le temps que dans l'espace, des modalités concrètes d'exercice des privatisations paraissent conférer au phénomène un contenu hétérogène et même discordant. Pourtant, les traits communs mais aussi les différences notoires que l'on observe d'un pays à l'autre, d'une période à l'autre, prennent toute leur signification si on les resitue par rapport aux mutations structurelles de ces économies et aux évolutions des politiques économiques qui y sont conduites.

Telle sera donc notre démarche. Nous mènerons de la sorte une rapide analyse du processus de privatisation durant la période allant de l'après-guerre au début des années soixante-dix (A) avant d'étudier plus précisément les privatisations réalisées depuis l'avènement de la crise économique (B).

\section{A - Les privatisations des trente glorieuses}

Durant les trente glorieuses, l'attitude des autorités publiques européennes à l'égard de leur patrimoine s'est sensiblement modifiée. Alors que, dans l'aprèsguerre, le développement de l'intervention publique directe est, presque partout, à l'ordre du jour 1), la situation va rapidement évoluer pour déboucher sur des privatisations 2 ).

\section{1) L'après-guerre}

Les privatisations ne sont pas d'actualité dans l'après-guerre. Un peu partout en Europe, on assiste au contraire à un renforcement voire à une extension très conséquente des secteurs publics productifs. En France, en Grande-Bretagne et en Autriche des nationalisations d'une ampleur sans précédent sont réalisées, notamment dans les secteurs de l'énergie et des transports (13). En RFA et en Italie, l'heure est à la recomposition et à la consolidation des secteurs publics hérités des régimes précédents et de l'économie de guerre.

En fait, le secteur public se trouve presque partout au centre du dispositif mis en place par les autorités pour reconstruire et relancer rapidement l'économie. L'interventionnisme public direct est donc de mise, tout particulièrement d'ailleurs dans les secteurs de base de l'économie, les entreprises publiques y étant le mieux à même de réaliser les investissements massifs désirés.

(13) Cf. « Le dossier des nationalisations ", Le Monde, Dossiers et Documents, supplément aux dossiers et documents du Monde, novembre 1977, pp. 75-76 et J.-C. ASSELAIN, « Nationalisations d'hier et d'aujourd'hui ", L'histoire, $\mathrm{n}^{\circ} 37$. 
Mais si, durant cette période, la publicisation d'actifs, par nationalisation ou croissance interne des secteurs publics, est manifeste, cela n'en ouvre pas moins l'ère des privatisations de par l'importance même prise par les patrimoines publics.

\section{Les privatisations des années de forte croissance et d'ouverture}

La forte croissance et l'ouverture qu'ont connues les économies libérales européennes à partir des années cinquante constituent les déterminants essentiels du changement général d'attitude des autorités publiques à l'égard de leur patrimoine productif et des privatisations qui en émanent.

Il ne se produit pourtant pas de remise en cause radicale de l'appropriation publique. Les préoccupations majeures des autorités publiques se déplacent progressivement pour se porter sur l'adaptation et le développement des activités industrielles concurrentielles, à prédominance privée (14).

Ces priorités nouvelles vont, le plus souvent, amener les États à faire jouer aux secteurs publics une fonction d'assistance au développement de l'activité privée. La privatisation par différenciation subordonnée prend alors consistance et ampleur en Europe. La réalisation d' " effets d'environnement " par les secteurs publics va être recherchée et se concrétiser par des compressions tarifaires. Ce sera notamment le cas au Royaume-Uni durant les périodes 1965-67 et 1970-73 (15) et en France avant 1967 (16). Le développement de la privatisation par différenciation va également s'effectuer - mais de manière beaucoup plus ponctualisée - à travers l'importante progression de l'économie mixte, celle-ci permettant d'opérer des transferts de ressources financières et des apports en capital public très sélectifs. La spectaculaire extension du « système des participations publiques ", notamment dans des pays comme l'Espagne (avec l'INI) et surtout l'Italie (avec le " modèle IRI ») (17), montre à quel point l'économie mixte peut constituer le support de politiques incitatives et de transfert (18).

Il ne faudrait cependant pas croire que les développements très conséquents de la filialisation publique observés à cette époque dans la quasi-totalité des économies d'Europe occidentale (19) relèvent de la privatisation par différenciation. Ils

(14) Pour reprendre la terminologie de Y. MORVAN, les « tactiques d'action " et en particulier de "substitution », qui étaient prédominantes, tendent à céder la place aux « tactiques d'environnement ». Cf. Y. MORVAN, «La politique industrielle française depuis la libération : quarante années d'intervention et d'ambiguïtés ", Revue d'économie industrielle, $n^{\circ} 23,1^{\text {er }}$ trimestre 1983, p. 21 .

(15) Cf. F.-E. DANGEARD, « Nationalisations et dénationalisations en Grande-Bretagne », Notes et études documentaires, La documentation française, $n^{\circ} 4739-4740,14$ novembre $1983, p$. 108 .

(16) Le rapport Nora dénonçait l'inefficacité des « transferts aveugles » opérés par les entreprises publiques françaises. Cf. Rapport sur les entreprises publiques, Groupe de travail du conseil interministériel des entreprises publiques, avril 1967, La documentation française, 1967.

(17) Le Monde, "Le Dossier des nationalisations », op. cit., pp. 78-79.

(18) Voir sur les notions de politiques réglementaire, de transfert, incitative : J. de BANDT, «La politique industrielle : réponse de l'État-Nation à la crise ? ", $R E I, \mathrm{n}^{\circ} 23$, op. cit., pp. 42-49.

(19) Cf. L. RAPP, Les filiales des entreprises publiques, Thèse en droit, Toulouse, 1982, introduction. 
procèdent également, pour une large part, de l'assimilation comportementale. L'ouverture de ces économies a très sensiblement avivé la concurrence y sévissant. Dans ces conditions, les directions d'entreprises publiques ont pu, surtout en secteur concurrentiel, faire valoir auprès des autorités la nécessité d'adapter leurs structures (voire leurs comportements) pour les rapprocher de celles de leurs concurrents privés.

Cette logique d'adaptation va même conduire certains États à chercher à pousser plus loin l'assimilation, en procédant à des dénationalisations. Ce sera le cas - sans grand succès, il est vrai - en RFA (20), au Danemark (21) ou encore au Royaume-Uni (22) (les motivations doctrinales ayant également joué un rôle non négligeable pour cette dernière nation).

L'avènement de la crise économique va cependant provoquer de profonds bouleversements quant à l'attitude des autorités publiques à l'égard de leur patrimoine et de sa privatisation. Les implications actuelles de telles mutations appellent une analyse détaillée.

\section{B - Privatisations et crise économique}

Les implications de la crise économique sur les secteurs publics européens furent immédiates et brutales. Dès 1974, la plupart d'entre eux va jouer un rôle contracyclique de grande ampleur dont il convient de préciser les conséquences en matière de privatisation 1). Cette « mise à contribution » massive des secteurs publics ne va pourtant pas pouvoir durer, conduisant à une phase nouvelle de privatisation 2).

\section{Mise à contribution des secteurs publics et politiques contracycliques}

La mise à contribution massive des entreprises publiques européennes leur causa des difficultés financières d'une ampleur sans précédent.

Au Royaume-Uni, la rentabilité des entreprises publiques (hormis British Gas et British Telecom) s'est fortement dégradée et ceci dès les prémices de la crise. A compter de 1971, la rentabilité hors subventions des industries nationalisées devient négative avec des minima en 74-75 et 79-80. En effet, malgré un ambitieux programme de nationalisation du parti travailliste, qui en faisait le régénérateur de l'économie, « bien loin d'étendre les participations de l'État dans les secteurs de pointe (...), le National Enterprise Board se vit contraint d'intervenir de façon croissante en faveur d'entreprises en faillite et de secteurs en déclin » (23). Les entreprises publiques monopolistiques furent de plus soumises à de nombreuses compressions tarifaires ce qui limita leur capacité d'investissement.

(20) Cf. "Le dossier des nationalisations ", Le Monde, op. cit., p. 75.

(21) Cf. Centre européen de l'entreprise public, $L$ 'évolution des entreprises publiques dans l'Europe des neuf », Édition du CEEP, Bruxelles, mai 1973, p. 45.

(22) Cf. F.-E. DANGEARD, « Nationalisations et dénationalisations en Grande-Bretagne, op. cit., pp. 34-35.

(23) F-E. DANGEARD, op. cit., pp. 26-27. Voir également J.-J. SANTINI, « Les privatisations à l'étranger ", Notes et études documentaires, $n^{\circ} 4821$, p. 34. 
En Italie, les difficultés financières sont plus évidentes encore. L'État a dû octroyer au secteur public près de 145 milliards de francs de 1973 à 1985 (24), ayant servi pour près de trois quarts à apurer les pertes. Les entreprises publiques sont alors victimes de l'obligation de rachat de sociétés en difficulté (25), du clientélisme électoral, et d'une "répartition politique 》 des postes à responsabilité.

De même, on a assisté en Espagne, entre 1975 et 1983, à la reprise publique d'entreprises en difficulté dans l'alimentation, le textile, la métallurgie et les transports maritimes. Le rapport rentabilité/fonds propres de l'INI est, en 1980, de - $0,1 \%$ alors qu'il aurait été, sans ces reprises, de $1,78 \%$. Plus généralement, les pertes des entreprises publiques non financières sont passées de $0,3 \%$ du PIB en 1977 à $1,6 \%$ en $1983(26)$.

En Autriche, il en fut ainsi mais plus tardivement. Les nationnalisées ont absorbé près de 32 milliards de francs de subventions entre 1981 et 1987, « le gouvernement ayant exigé que les objectifs particuliers des entreprises publiques soient subordonnées à ses objectifs de politique économique " (27). Ces contraintes ont notamment concerné l'emploi, l'investissement, l'aide aux entreprises en difficulté.

Le cas français est quant à lui plus complexe (car polymorphe) et plus original. La publicisation d'actifs y joue également un rôle important mais sur des bases foncièrement différentes (28). De 1973 à 1981, les entreprises publiques monopolistiques - notamment des secteurs électronucléaires et des télécommunications (29) - sont amenées par l'État à réaliser des investissements massifs débordant toute rationalité micro-économique et débouchant sur un endettement considérable. Cet effort fut relayé par le secteur public concurrentiel, suite (et grâce) aux nationalisations de 1982-83 (30). La situation financière des entreprises publiques s'aggrave cependant fortement jusqu'en 1984 (31), obligeant notamment l'Etat à leur octroyer près de 50 milliards de francs de fonds propres de 81 à 85 .

(24) L'IRI et l'ENI accumulaient en 1983 près de 140 milliards de francs de pertes.

(25) L'ENI “ hérite " d'unités textiles, l'IRI devient une " poubelle de canards boiteux ".

(26) Cf. R. MYRO, Privatization, the Spanish experience, Rapport aux ministres, Bruxelles, janvier 1987 , p. 5.

(27) Cf. A. BACHLEITNER, B. GENSER, R. MEISSL et A. VAN DER BALLEN, « Offentliche unternehmen in Österreich ", Annales de l'économie publique, sociale et coopérative, septembre 1985 ou encore Problèmes économiques, 23 avril 1986, $\mathrm{n}^{\circ} 1971$.

(28) Sauf dans le secteur des transports maritimes. Cf. P. BANCE, thèse précitée, pp. 129-131.

(29) On assiste ainsi, durant la période 1975-1981, à « une très forte progression des capitaux publics " dans les secteurs nucléaire, pétrole et transports. Cf. LEREP, «Propriété et pouvoir dans l'industrie ", 1, Notes et études documentaires, $\mathrm{n}^{\circ} 4832,1987-7$, p. 49.

(30) Cf. J.-F. ESCARMELLE, L'État industriel dans les politiques de sortie de crise, Cabay, 1986, pp. $175-179$.

(31) La détérioration des comptes de Renault et de la sidérurgie y est cependant pour beaucoup. 
En ces premières années de crise, les secteurs publics sont donc soumis à des pressions considérables des États qui conduisent à leur extension et à la publicisation de leurs comportements. Dans le cadre des orientations étatiques, ils assument alors, entres autres missions, des fonctions d'assistance au redéploiement et de soutien sélectif aux capitaux privés tout en générant des effets d'environnement. La privatisation par différenciation subordonnée prend donc une ampleur inégalée (32).

Les difficultés financières croissantes qui résultèrent alors, pour les entreprises publiques, de cette mise à contribution massive leur interdisaient cependant de poursuivre leur effort, du moins sans aides de l'État (33). Or, les autorités publiques ont, à partir de la fin des années soixante-dix, exprimé des réticences de plus en plus fortes pour couvrir les pertes des entreprises publiques, leurs objectifs premiers devenant la réduction du déficit budgétaire et des prélèvements obligatoires. Les déficits des entreprises publiques ne manquèrent pas, par ailleurs, d'alimenter les critiques radicales à l'égard de l'appropriation publique et contribuèrent à faire passer dans l'opinion l'idée, simpliste, de carences intrinsèques fondamentales la concernant.

L'effort des entreprises publiques ne pouvant plus se poursuivre ni sur ressources propres ni sur apports étatiques, l'ère de la privatisation par assimilation était ouverte.

\section{Les privatisations des années quatre-vingt}

Si l'épuisement des vertus contra-cycliques des secteurs publics va amener, à partir du début des années quatre-vingt, les États européens à s'engager dans la voie de la privatisation par assimilation de leur patrimoine (a), les convergences internationales ne sont pas totales : le processus de privatisation par assimilation présente une double nature (b).

\section{a) La montée de la privatisation par assimilation comportementale}

Les États vont fréquemment laisser carte blanche aux directions des groupes publics pour redresser la situation financière, ce qui va rapidement produire ses effets, la conjoncture économique étant, de surcroît, assez favorable.

En Italie, le groupe ENI renouait ainsi dès 1985 avec les bénéfices $(2,18$ milliards de francs) et l'IRI, en 1986, (1,68 milliard). Le taux d'endettement (34) de l'IRI passait de 103 à $73 \%$ de 1982 à 1986 et l'ENI multipliait dans le même temps par quatre son cash flow. Depuis 1982, ces groupes ne se sont plus portés acquéreurs d'entreprises en difficulté et ont redressé ou vendu celles qu'ils détenaient.

(32) Cela n'exclut bien entendu aucunement d'autres modalités de mise à contribution des patrimoines publics (autres formes de publicisation comportementale : maintien de sureffectifs pour lutter contre le chômage, endettement extérieur pour défendre la monnaie...).

(33) L'État espagnol fit cet effort jusqu'en 1983 mais cela contribue à cette date, en tenant compte des subventions d'exploitation et des sommes allouées pour éponger les pertes, à $36 \%$ du déficit des administrations publiques. Cf. R. MYRO, op. cit., p. 6.

(34) Rapport endettement sur chiffre d'affaires. 
De sévères dégraissages ont eu lieu, les effectifs de l'IRI ayant ainsi chuté de $16 \%$ depuis 1980 (35).

En Espagne, l'INI a également réalisé des restructurations et des liquidations à partir de 1984. Une reconversion des secteurs en déclin a été entamée et les entreprises déficitaires du groupe ont connu de fortes compressions d'effectifs (36).

Il en est de même au Royaume-Uni de 1979 à 1983 : « le nombre de salariés est passé de 186 à 81 mille dans la sidérurgie, de 177 à 107 mille chez British Leyland, de 56 à 35 mille chez British Airways, etc. " (37). Les profits des entreprises publiques industrielles se sont ainsi accrus de $20 \%$ par an de 80 à 85 .

L'autonomie de gestion accordée aux groupes publics français, institutionnalisée par les contrats de plan, a permis leur redressement financier. Si l'on excepte Renault, Usinor et Sacilor, les bénéfices publics ont été portés de 0,389 à 12,7 milliards de francs de 1983 à 1985 (38).

\section{b) Les deux voies de la privatisation}

Les convergences internationales observées en matière de privatisation ne doivent pas cacher les deux philosophies opposées qui la sous-tende : la reconstitution (b1) et la cessation d'activité publique (b2).

\section{b1. La privatisation-reconstitution}

Elle concerne toute mesure de privatisation dont l'objet est de restaurer la capacité des entreprises publiques de répondre aux contraintes de l'État. Contrairement à la nationalisation qui étend la capacité d'intervention publique par élargissement du secteur public, la privatisation-reconstitution est une stratégie visant à redonner vigueur à des groupes ou entreprises y figurant déjà pour leur permettre, à terme, d'assumer de nouveau des missions de politique économique.

L'Italie est sans nul doute engagée dans cette voie depuis 1983. Les groupes publics n'ont nullement cédé les activités considérées comme stratégiques, se séparant par contre de filiales non stratégiques déficitaires. Seules l'efficacité et le rétablissement financier des groupes publics sont alors recherchés. L'État ne remet

(35) Concernant l'amélioration de la situation financière des entreprises publiques en Italie, cf. « Les secteurs privé et public en Italie : sous le signe du redressement ", Problèmes économiques, ${ }^{\circ} 1992$, $1^{\text {er }}$ octobre 1986, pp. 2-7.

(36) Seat (automobile) Iberia, (transports aériens), Bazán (matériel militaire) et Babcok sont particulièrement concernés. Le déficit de l'INI a ainsi pu être ramené de 11 à 8,4 milliards de francs de 1984 à 1986. Cf. J.-J. SANTINI, op. cit., p. 105.

(37) Cf. J.-J. SANTINI, op. cit., p. 37.

(38) Les résultats nets du secteur public tout entier (y compris Renault et la sidérurgie) sont passés de -12 à -7 milliards de francs de 83 à 85 . Voir sur la privatisation comportementale des groupes publics français après 1982 : E. COHEN, " Nationalisations : une bonne leçon de capitalisme ", Politique économique, $\mathrm{n}^{\circ} 1$ (nouvelle série), avril 1986. Voir également (notamment sur la question du financement) : G. PRACHE, « Les entreprises publiques et le marché des fonds propres, entre nationalisations et privatisations "(1982-1986) ", Analyse financière, 1er trimestre 1987. 
aucunement en cause le principe de l'appropriation publique, la nationalisation elle-même n'étant pas proscrite dans une optique de restructuration.

Il en est ainsi en Espagne (39). « Pour l'INI et Patrimonio, les entreprises privatisables ne doivent pas avoir de raisons économiques de résider dans le secteur public parce que leurs activités ne complètent pas utilement les filières de production des groupes publics ou bien parce qu'elles ne représentent pas un intérêt national stratégique » (40). De plus, les dénationalisées furent, le plus souvent, vendues tout en restant déficitaires, ce qui a évité aux groupes publics de procéder à de coûteuses opérations de redressement.

Ces privatisations, par assimilation comportementale ou dénationalisation, procèdent donc de la reconstitution de l'instrument dynamisateur et régulateur qu'est le secteur public. Nous parlerons alors de mise en sommeil forcée du patrimoine public en tant qu'outil de politique économique.

\section{b2. La privatisation-cessation d'activité publique}

Le Royaume-Uni et la France (en 1987) se sont, quant à eux, engagés dans des politiques de dénationalisation massive, se démarquant d'ailleurs très nettement de l'ensemble des autres pays européens de par l'ampleur de leurs programmes et réalisations. Au delà des considérations budgétaires et fiscales, ces opérations relèvent d'une opposition doctrinale très marquée vis-à-vis de l'appropriation publique, tout particulièrement en secteur concurrentiel.

L'assimilation comportementale s'analyse, dans ce cadre, comme une phase préliminaire à la transmutation la plus radicale des capitaux publics : la dénationalisation. La bonne rentabilité des groupes (ou actifs) que l'on entend transférer au privé est en effet une condition nécessaire au bon déroulement d'une politique de dénationalisation massive, ne serait-ce que pour la rendre attractive aux yeux des petits porteurs.

Il n'y pas alors pour autant renonciation totale des autorités à soumettre le secteur public à leurs orientations de politique économique. La mise à contribution - et notamment la privatisation par différenciation - peut en effet porter sur un sous-ensemble d'entreprises publiques non concerné par la dénationalisation. Les entreprises publiques monopolistiques sont particulièrement visées. En France, EDF s'est ainsi vu imposer, en 1987-88, des réductions tarifaires successives alors que son contrat de plan était censé lui garantir des augmentations de prix (41).

(39) Il en est ainsi malgré des déclarations d'hommes politiques de premier plan marquant un attachement à l'initiative privée. Le premier ministre espagnol, F. GONZALEZ, déclarait le 3 septembre 1986 : « Je crois que dénationaliser et donner de l'initiative au secteur privé est une attitude de progrès. Par contre, penser que le progrès signifie nationalisation et contrôle de l'économie est une erreur conceptuelle $»$.

(40) Cf. J.-J. SANTINI, op. cit., pp. 110-111.

(41) Précisons cependant que les conditions d'approvisionnement étaient devenues plus favorables, suite notamment à la date du cours du dollar. Il n'en reste pas moins que, pour les dirigeants d'EDF, cela contribue à des retards dans les investissements visant à améliorer le réseau. 


\section{CONCLUSION}

Le concept de privatisation est riche en déterminations multiples. La confusion, très répandue présentement, avec le concept de dénationalisation est révélatrice d'une focalisation du débat sur la seule logique de la substitution du secteur privé au secteur public. Elle tronque en fait la réalité profonde du phénomène.

Beaucoup plus qu'une pratique à la mode dans les années quatre-vingt, les privatisations du patrimoine public s'analysent dans les économies libérales comme un processus contradictoire dont le caractère évolutif tient aux mutations structurelles de ces économies, à la nature des politiques économiques qui y sont conduites et aux difficultés rencontrées par les secteurs publics pour répondre aux missions de politique économique qui leur sont confiées.

L'analyse rétrospective montre cependant que, si l'histoire ne se répète pas, de profonds bouleversements (pour ne pas dire revirements) peuvent se produire brutalement quant à l'attitude des États à l'égard de leur patrimoine et de sa privatisation. La privatisation par assimilation s'est ainsi déjà soudainement effacée devant la privatisation par différenciation et inversement. Bien souvent les vérités du jour ne sont pas celles du lendemain. Loin de nous donc l'idée que les privatisations des années quatre-vingt, marquées par l'assimilation des unités publiques au secteur privé voire par la dénationalisation, soient un mouvement de fond rendu irréversible par l'ouverture croissante des marchés et l'intensification de la concurrence (abstraction faite cependant de la réalisation du marché unique européen).

Il n'est d'ailleurs pas même possible de considérer que les privatisations d'aujourd'hui sont fondamentalement semblables d'un pays à l'autre. Les politiques actuelles de privatisation, et en particulier de dénationalisation, renvoient en effet à des philosophies antagoniques : les unes marquant une opposition de principe à l'appropriation publique, les autres visant à redonner force au secteur public pour l'impliquer de nouveau dans des missions de politique économique.

Autant de raisons qui conduisent à prôner, au moins pour le discours scientifique, la rigueur dans l'approche conceptuelle ainsi que la circonspection dans l'analyse comparative et prospective. 